\title{
ESTIMACIÓN DE PARÁMETROS HIDRODINÁMICOS EN UN ACUÍFERO VOLCÁNICO LIBRE FRACTURADO EN COSTA RICA. PARTE I
}

\author{
ESTIMATION OF HYDRODINAMIC PARAMETERS IN A VOLCANIC \\ FRACTURED PHREATIC AQUIFER IN COSTA RICA. PART I
}

\author{
Julio Macías ${ }^{1}$, Asdrúbal Vargas ${ }^{2 *} \&$ Francisco Frutos $^{3}$ \\ ${ }^{1}$ Consultor Geomecánica \\ ${ }^{2}$ Instituto Costarricense de Electricidad, Apdo. 2300-Sabana Norte, \\ San José Costa Rica \\ ${ }^{3}$ Escuela de Física, Universidad de Costa Rica \\ *Autor para contacto: avargass@ice.go.cr
}

(Recibido: 15/06/2015; aceptado: 10/03/2016)

\begin{abstract}
Estimated values for transport parameters are reported for the conditions of a volcanic fractured phreatic aquifer in Costa Rica. Three salt-tracer tests performed under natural gradient conditions were interpreted with the aid of single porosity advective-dispersive analytical solutions for pulse and instantaneous injections. Field breakthrough curves showed a clear asymmetric shape, very common for fractured systems. Applied analytical solutions allowed to gain insights into the understanding of the transport processes involved during solute transport in fractured systems, but did not allow for the reproduction of the observed asymmetrical shape. Flow parameters were also obtained (saturated hydraulic conductivity; effective porosity and Darcy's flux). The calculated values are within the range of values reported in the literature for fractured aquifers.

Keywords: Fractured aquifer, analytic model, tracer test, spring, hydrogeology.

RESUMEN: En este trabajo se presenta una estimación del valor de los parámetros de transporte de solutos conservativos para un acuífero volcánico fracturado, obtenida a partir de la interpretación de tres ensayos de trazador inyectados como pulsos instantáneos y analizados por medio de modelos advectivos-dispersivos unidimensionales de porosidad simple. El trazador fue vertido en una perforación exploratoria, y simultáneamente se monitoreó la conductividad eléctrica en el fluido descargado por un manantial ubicado cerca del maar de la Laguna Hule, que abastece la comunidad de San Miguel de Sarapiquí. Los valores de conductividad eléctrica fueron transformados a concentración, para obtener así curvas de llegada del soluto hasta el manantial. Los resultados muestran un claro comportamiento asimétrico en la curva de concentración, típico de sistemas fracturados. Para el análisis de los ensayos de trazador se realizó un ajuste numérico entre la curva de concentración de campo (o curva de llegada) y la solución analítica advectiva-dispersiva para inyección instantánea. Esta solución no permitió reproducir la asimetría observada en los
\end{abstract}

MACÍAS, J., VARGAS, A., FRUTOS, F., 2016: Estimación de parámetros hidrodinámicos en un acuífero volcánico libre fracturado en Costa Rica. Parte I.- Rev. Geol. Amér. Central, 55: 167-183, DOI: 10.15517/rgac.v55i0.27073 
datos de campo, sin embargo permitió además de entender los mecanismos de transporte actuantes obtener valores de dispersividad los cuales se encuentran dentro del rango reportado en la literatura mundial.

Debido a que las propiedades de flujo del acuífero no fueron caracterizadas explícitamente durante los ensayos de trazador, se calcularon los valores de conductividad hidráulica (k), velocidad de Darcy (vd) y la porosidad efectiva a partir de las velocidades de poro obtenidas en el ajuste de las curvas de llegada del soluto. Los valores obtenidos se encuentran dentro del rango de valores obtenidos por medio de ensayos de bombeo en otros acuíferos fracturados.

Palabras clave: Acuífero fracturado, modelo analítico, ensayo trazador, manantial, hidrogeología.

\section{INTRODUCCIÓN}

En las principales ciudades de Centroamérica, más del $50 \%$ del abastecimiento de agua potable proviene de mantos acuíferos volcánicos localmente fracturados, desarrollados en lavas masivas, lavas porosas, tobas y piroclastos (Losilla et al., 2001). Las propiedades de flujo son generalmente conocidas para los acuíferos más importantes, sin embargo las propiedades de transporte de solutos son desconocidas, sea por la escasa inversión en la investigación de este tema o porque esa información queda restringida a proyectos de interés privado.

Los ensayos de trazador en campo constituyen la mejor manera de obtener dichas propiedades. Estos consisten en la inyección de una masa en un punto y su posterior identificación en términos de concentración o color en otro punto localizado aguas-abajo en el sentido del flujo. Dichos ensayos pueden ser realizados bajo diversas condiciones, a saber: campo o laboratorio, bajo gradiente natural o gradiente impuesto, con patrones de flujo radial o lineal $\mathrm{y}$, con inyección continua o instantánea (Ptak et al., 2004). Los trazadores ideales son aquellos que no reaccionan con el medio circundante y que no se degradan durante el transporte. Los más comunes son: sales de cloruros (v.g $\mathrm{NaCl}$ ) o bromuros (v.g. $\mathrm{NaBr}$ ), tintes, isótopos ambientales y virus.

El procedimiento convencional de análisis de un ensayo de trazador consiste inicialmente en la caracterización del régimen de flujo. Esto es, la determinación de la permeabilidad, porosidad del medio, así como el gradiente hidráulico y la velocidad de flujo macroscópica o a nivel de poros. Posteriormente, se procede a la determinación de los parámetros de transporte. Dependiendo de los procesos involucrados en el trasporte puede requerirse la determinación de más de un parámetro. Para este procedimiento se realiza un ajuste numérico entre la curva de concentración de campo (o curva de llegada medida en el punto de observación) y, una curva teórica obtenida a través de una solución analítica o numérica (Escuder et. al, 2009). Diversas soluciones han sido propuestas para modelar el transporte de trazadores en dependencia al tipo de material, patrón de flujo o esquema de inyección. Algunas de estas soluciones pueden ser revisadas en Tsang et al. (1981), Custodio \& Llamas (1983), Maloszewski \& Zuber (1990), y Fetter (1993).

En el año 2001, como parte de los estudios de evaluación del impacto ambiental del Proyecto Hidroeléctrico Cariblanco, se realizaron una serie de estudios con el objetivo de analizar la posible afectación del manantial San Miguel (el cual alimenta el acueducto de San Miguel de Sarapiquí) por los trabajos de excavación para la implantación del embalse Cipreses. Como parte de dichos estudios, se realizaron tres ensayos de trazador entre los meses de octubre y noviembre, orientados principalmente a evaluar la conectividad entre la zona del embalse y los diferentes acuíferos del lugar, pero no orientados a la obtención de parámetros de transporte. Los resultados de dichos análisis fueron presentados por Vargas \& Fernández (2002). Estos autores indican que el manantial San Miguel es producto de la descarga de un acuífero superficial de tipo libre desarrollado en 
lavas con porosidad primaria por vesículas (poros, sin embargo no conectados entre ellos) y secundaria por fracturas, es decir un acuífero fracturado.

Los datos de los ensayos de trazador referidos no habían sido interpretados hasta la fecha para obtener parámetros hidrodinámicos representativos de este tipo de acuíferos. Sin embargo, debido a la carencia de esta información a nivel centroamericano, se consideró por parte de los autores de este trabajo como una oportunidad para contribuir al conocimiento sobre este tópico.

Luego de una revisión inicial de la información disponible y considerando el hecho de que la recopilación de la información en campo no estuvo orientada a la simulación de las curvas de llegada, se concluyó que era posible estimar valores para los parámetros de transporte; sin embargo se requeriría de un procedimiento no convencional de análisis. La metodología aplicada para dicho análisis fue la siguiente: (1) como primer paso, se realizó un ajuste de las curvas de llegada (en unidades de concentración normalizada) con modelos unidimensionales (1D) de porosidad simple para inyecciones de tipo instantánea y por pulso, esto es, idealizando el medio fracturado como un medio continuo equivalente. Este análisis se realizó para modelos 1D por contar solamente con un punto de observación; (2) paralelamente se procedió a calcular la permeabilidad del acuífero a partir de los datos estimados de caudal de la naciente San Miguel y de los datos de campo registrados durante el primer ensayo de trazador, (3) con la permeabilidad así obtenida y en conjunto con la velocidad de poros calculada a través del ajuste de las curvas de llegada en el paso 1, se calcularon la porosidad y velocidad de Darcy, es decir, se completó la caracterización del régimen de flujo para las condiciones del primer ensayo. (4) A partir de la permeabilidad y porosidad obtenidas junto a las velocidades de poro resultantes del ajuste (1) se completó la caracterización del flujo para las fechas de los ensayos siguientes. (5) Conocidas las condiciones de flujo para los tres ensayos se procedió a realizar un nuevo ajuste de las curvas de llegada mediante el uso de un modelo de simulación más complejo (modelo MIM) que incorporara el efecto del fracturamiento del acuífero.
En este documento se presentan los resultados obtenidos hasta el punto 4 de la metodología arriba descrita. Es decir este documento se centra en los ajustes realizados con modelos de porosidad simple para un medio continuo equivalente. Los resultados incluyendo los ajustes con el modelo MIM así como la validación de los parámetros obtenidos serán presentados en una publicación posterior.

La utilidad de esta investigación está centrada en como la interpretación de ensayos de trazador pueden permitirle al investigador ambiental obtener parámetros hidrodinámicos de acuíferos fracturados para evaluar el potencial de contaminación de nacientes o captaciones de agua asociadas a este tipo de acuíferos por actividades antropogénicas (estaciones de servicio, rellenos sanitarios, actividades agrícolas, etc.).

\section{UBICACIÓN Y CONTEXTO HIDROGEOLÓGICO DEL MANATIAL SAN MIGUEL}

El área de estudio está localizada en la vertiente noroeste de la Cordillera Volcánica Central, en la hoja topográfica Poás (3346 I) en escala 1:50 000 , entre las coordenadas $248000-252500$ y 512 000-516 000 (Fig. 1). La elevación del terreno varía entre 800 m.s.n.m y 2000 m.s.n.m. En la figura 1 también se muestra la ubicación del manantial San Miguel (coordenadas planas 252188 latitud Norte y 514569 de longitud Oeste) en relación con la localización de los puntos de sondeo exploratorios 37PHC y $40 \mathrm{PHC}$, así como la ubicación del Embalse Cipreses.

El manantial San Miguel es un área de aproximadamente $4 \mathrm{~m}^{2}$ donde ocurre la descarga directa de un acuífero de tipo libre desarrollado en lavas basálticas fracturadas. Este acuífero presenta un espesor promedio de $24 \mathrm{~m}$ (detectado en el sondeo 37PHC) y se encuentra cubierto por una capa de coluvio de baja permeabilidad con espesor de $21 \mathrm{~m}$. En la figura 2 se muestra el núcleo de roca extraído de este acuífero donde se observan claramente al menos dos sistemas preferenciales de fracturas (inclinadas de poca abertura 


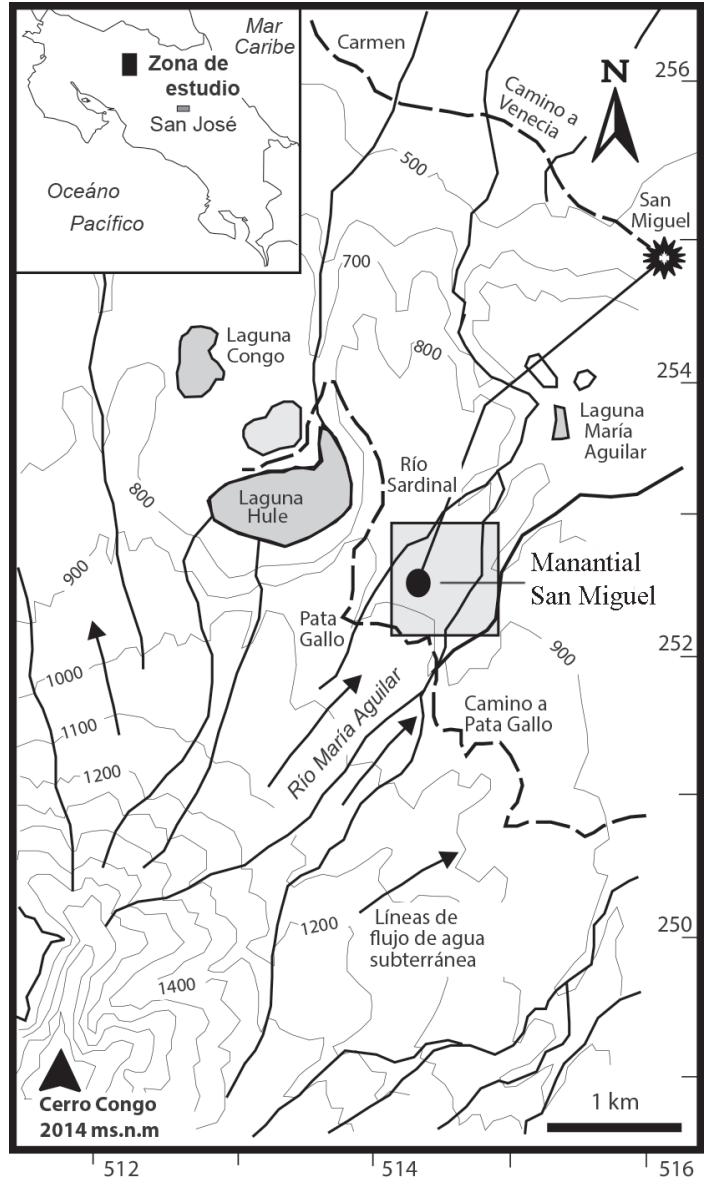

Fig. 1: Ubicación de la zona de estudio y del manantial San Miguel.

y subverticales abiertas) que facilitan el flujo de agua. Se interpreta que el origen de las fracturas inclinadas es debido posiblemente a un proceso tectónico de compresión horizontal, mientras que las fracturas subverticales fueron originadas por un proceso extensional posiblemente relacionado con el mismo régimen de esfuerzos que formó la laguna Hule.

De acuerdo con las descripciones de los núcleos estas lavas son correlacionables con los materiales lávicos originados en el Cerro Congo mencionados por Alvarado \& Salani (2004).

El acuífero se extiende longitudinalmente hasta aproximadamente 15 metros hacia el norte del manantial San Miguel (Fig. 3), y con una extensión lateral de al menos $200 \mathrm{~m}$ (Vargas \& Fernández, 2002). El nivel del agua subterránea

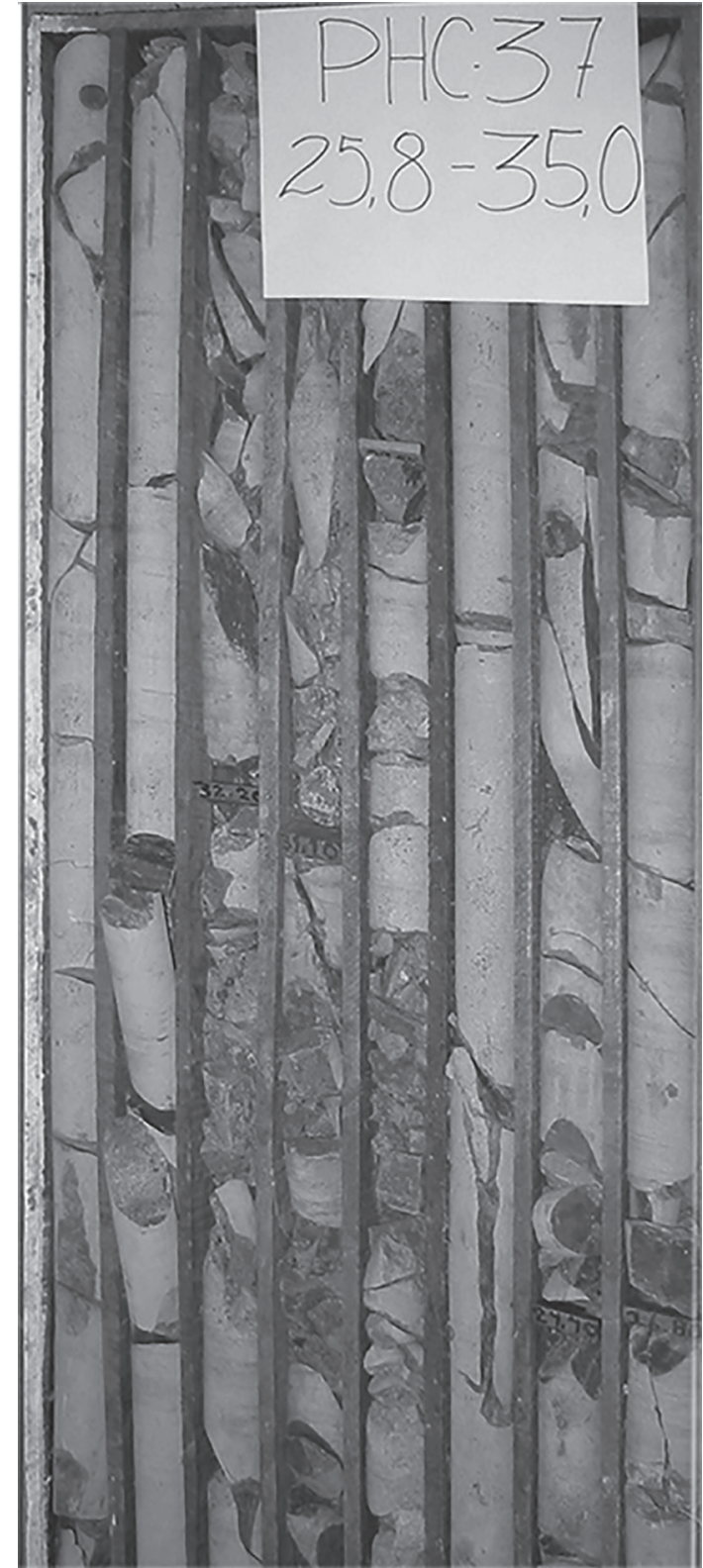

Fig. 2: Núcleo de roca obtenido en sondeo $37 \mathrm{PHC}$ para profundidades $25,8-35,0 \mathrm{~m}$.

se localizó a 27,3 m de profundidad en el sitio del sondeo 37PHC el día 11 de octubre de 2001 (elevación 867,7 m.s.n.m.), generando un gradiente hidráulico de $0,033 \mathrm{~m} / \mathrm{m}$, entre el sitio de sondeo y el manantial San Miguel (elevación 865,1 m.s.n.m.) para una distancia de $78,8 \mathrm{~m}$. 


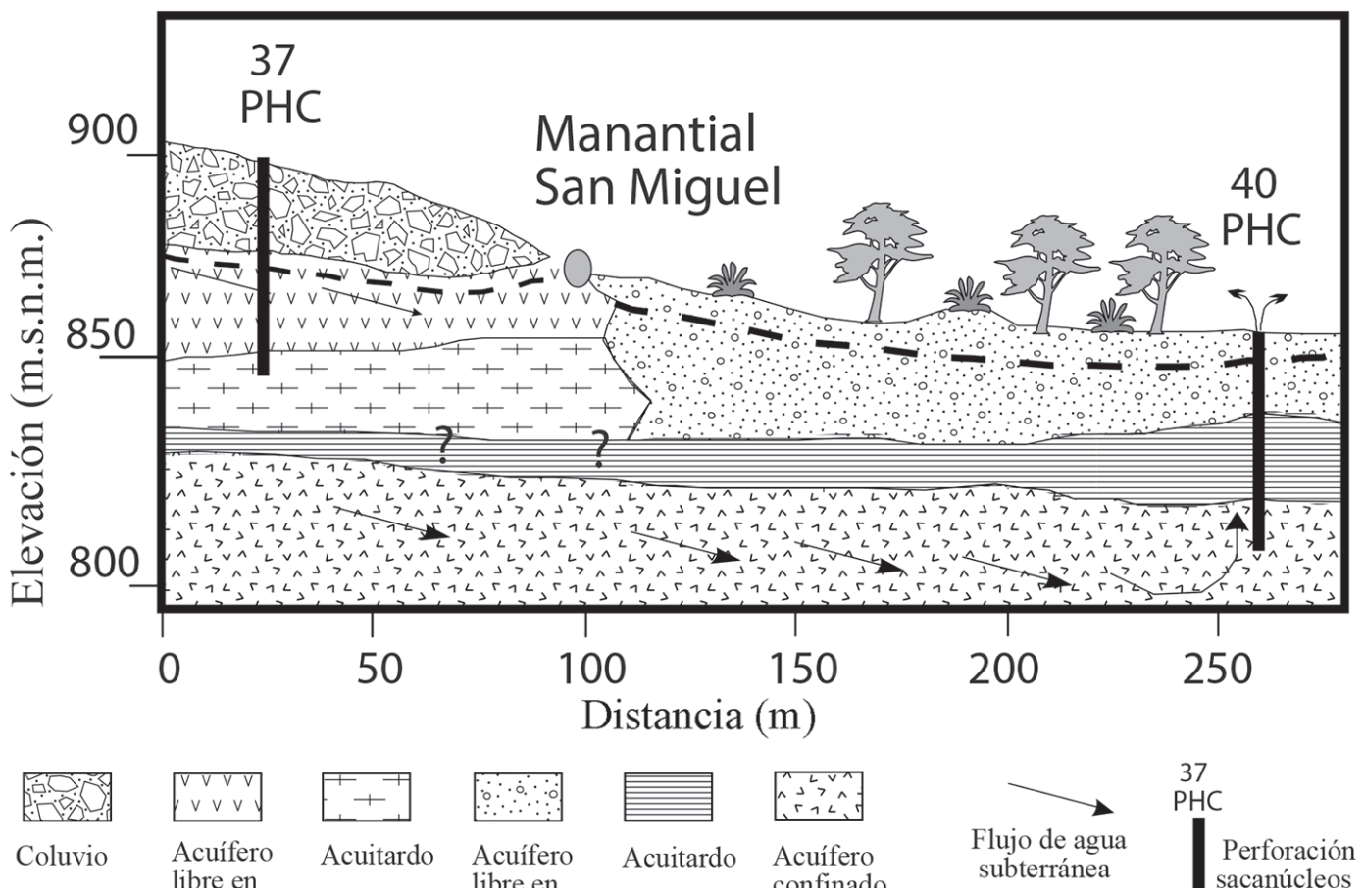

Fig. 3. Interpretación hidrogeológica de la zona de estudio.

\section{DESCRIPCIÓN Y RESULTADOS DE LOS ENSAYOS DE TRAZADOR}

Fueron realizados tres ensayos de trazador bajo condiciones de flujo en gradiente natural entre octubre y noviembre del año 2001. Se empleó sal común ( $\mathrm{NaCl}$-cloruro de sodio) como trazador por ser un soluto conservador no reactivo y especialmente por no representar amenaza para la salud humana. La solución de sal fue inyectada en el sondeo 37PHC (con diámetro de $0,07 \mathrm{~m}$ ), y la respuesta del acuífero fue monitoreada en el manantial San Miguel a través de la medición de la conductividad eléctrica con un conductivímetro de campo.

El día 11 de octubre del año 2001 por medio del Centro de Servicio de Auscultación de Obras del Instituto Costarricense de Electricidad (ICE) se procedió a realizar el primer ensayo (Ensayo 1), disolviéndose $45,5 \mathrm{~kg}$ de sal en un volumen de185 litros de agua. Esta solución fue inyectada en un período de 20 min de manera continua a través del uso de una manguera. Inmediatamente se midió la conductividad eléctrica del agua en el manantial San Miguel en lapsos de tiempo de 1 a 20 min durante 4,3 horas. Con el fin de corroborar la efectividad del primer ensayo se procedió a realizar un segundo ensayo el día 17 de octubre de 2001 (Ensayo 2). En este caso se disolvieron $90,1 \mathrm{~kg}$ de sal en un volumen de 185 litros de agua. La conductividad se midió en lapsos de tiempo de 0,5 a 10 min durante 6 horas. El día 1 de noviembre de 2001 se realizó un tercer ensayo (Ensayo 3), que de acuerdo con el reporte de campo se inyectó "con un volumen similar de solución al ejercicio anterior, pero con una solución saturada de cloruro de sodio". En este caso se registró la conductividad durante un lapso de 5 horas en intervalos de 1 a 6 min.

Los valores de conductividad eléctrica medidos durante los tres ensayos fueron transformados a valores de concentración de sal empleando una constante obtenida por medio de una calibración en laboratorio. Adicionalmente, estos valores fueron corregidos por la concentración de fondo, la cual fue monitoreada 
durante varios minutos previos al arribo del trazador al manantial. La concentración así obtenida se denomina concentración corregida, y cualquier variación de ella es producto del efecto de los procesos físicos actuantes durante el desplazamiento del trazador a través del acuífero.

En la figura 4 se presenta la concentración corregida para cada ensayo, en función del tiempo transcurrido desde el momento de la inyección. Dichas curvas representan la curva de llegada del trazador al manantial San Miguel. En la figura $5 \mathrm{se}$ muestran los mismos resultados pero normalizados al valor de concentración máxima o de pico registrada en cada ensayo. Esta normalización permite comparar directamente las curvas desde el punto de vista morfológico y analizarlas numéricamente sin importar las diferencias en las concentraciones de inyección para cada ensayo. Las curvas así obtenidas, a pesar de ser morfológicamente similares, indican diferencias en las condiciones de transporte para cada fecha de ensayo.

\section{Tiempos de arribo de la concentración máxima}

Los tiempos de arribo de las concentraciones de pico para los ensayos 1 a 3 fueron 3650 s, 8580 s y 8700 s respectivamente. Dichos tiempos corresponden con la primera detección de la concentración máxima. Es importante señalar que para el Ensayo 3 el muestreo de datos durante los 44 minutos previos a la llegada del valor pico se realizó a cada 1 min. Para el caso del Ensayo 2 el muestreo a cada $1 \mathrm{~min}$. se mantuvo hasta los 9 minutos anteriores del arribo del valor pico, y después la frecuencia de medición fue modificada. En este último caso existe un periodo de $7 \mathrm{mi}-$ nutos sin mediciones inmediatamente antes de la detección del pico.

De la figura 5 es claro el menor tiempo requerido durante el Ensayo 1 para la llegada de la concentración pico. Ese tiempo fue menor a la mitad del tiempo requerido para los ensayos 2 y 3 . El tiempo de arribo para estos dos últimos ensayos fue muy similar, presentándose una diferencia de $120 \mathrm{~s}$ entre ellos. A pesar de esta diferencia reducida en el arribo de las concentraciones de pico, la primera detección del trazador ocurrió a los 6000 $\mathrm{s}$ en el Ensayo 3, esto es $900 \mathrm{~s}$ más temprano que en el Ensayo 2. Es decir, aunque las concentraciones pico llegaron en aproximadamente el mismo tiempo, parte del soluto del Ensayo 3 llegó al manantial San Miguel más tempranamente.

El menor tiempo de arribo para el Ensayo 1 indica un mayor flujo de agua subterránea para esa fecha. Aunque los ensayos 2 y 3 se realizaron bajo condiciones de flujo muy similares, la detección más temprana durante el Ensayo 3 demuestra efectos dispersivos mayores actuando durante ese ensayo.

\section{Valor de concentración máxima o de pico}

Las concentraciones de pico obtenidas fueron $3,54 \mathrm{mg} / 1,10,62 \mathrm{mg} / 1$ y $186,27 \mathrm{mg} / \mathrm{l}$ para los ensayos 1, 2 y 3 respectivamente. Las diferencias entre estos valores son reflejo principalmente de las diferentes concentraciones de inyección empleadas para cada ensayo y de los efectos generados por la magnitud relativa de los procesos dispersivos y advectivos actuantes durante el transporte. Para los ensayos 1 y 2 se reportaron cantidades de sal inyectadas de 45,5 $\mathrm{kg}$ y $90,1 \mathrm{~kg}$. Sin considerar las posibles diferencias en la velocidad del agua subterránea para esas dos fechas de ensayo, la cantidad de trazador inyectado explica parcialmente la diferencia entre las concentraciones de pico medidas. Para los ensayos 2 y 3 , a pesar de mostrar un tiempo de arribo muy similar, se observó una diferencia muy importante en las concentraciones de pico. De acuerdo al reporte de campo, en este último ensayo se aplicó una solución salina saturada, sin embargo no se menciona explícitamente el valor de esa concentración. No se cuenta con mayor información para establecer realmente si dicha solución saturada corresponde con la misma concentración inyectada durante el Ensayo 2. La diferencia en las concentraciones de pico permite inferir que probablemente se empleó una concentración mayor. Para efectos de los análisis subsecuentes en este trabajo y debido a la carencia de información se asumió la misma concentración del Ensayo 2. 


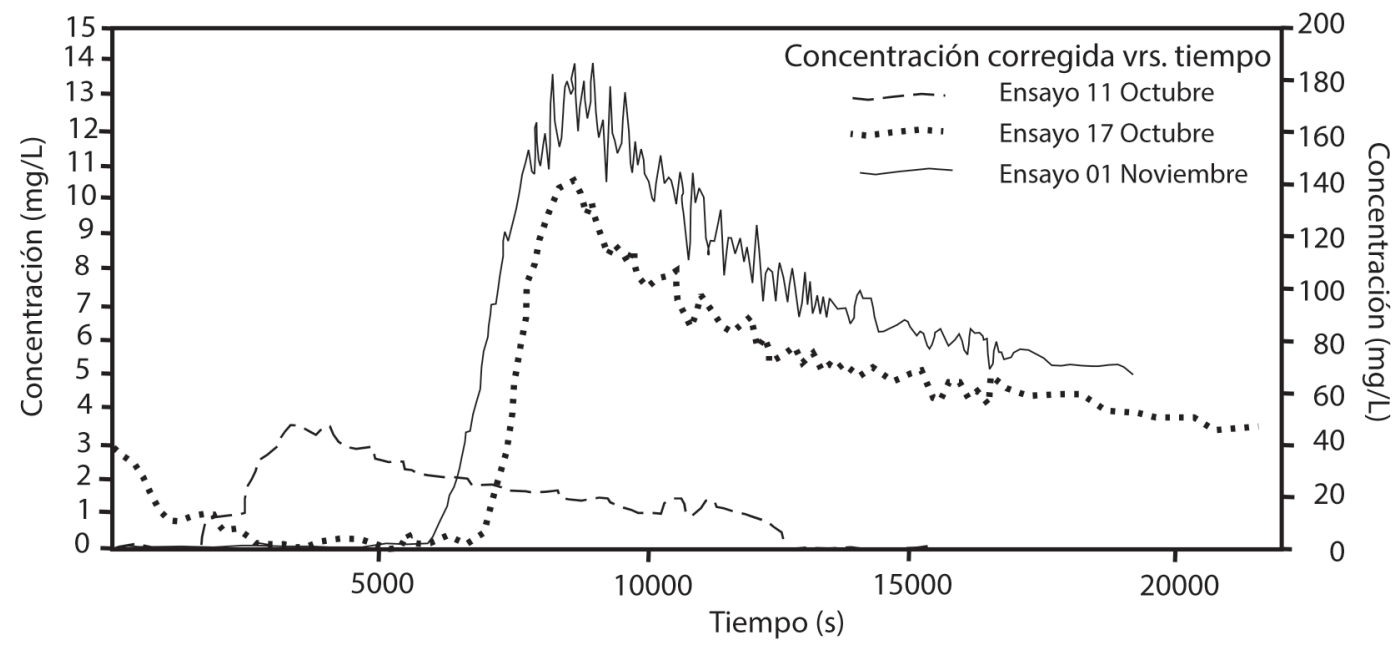

Fig. 4: Curva de llegada para cada ensayo de trazador (Concentración para el Ensayo 3 del 01 de noviembre de 2001en el eje a la derecha).

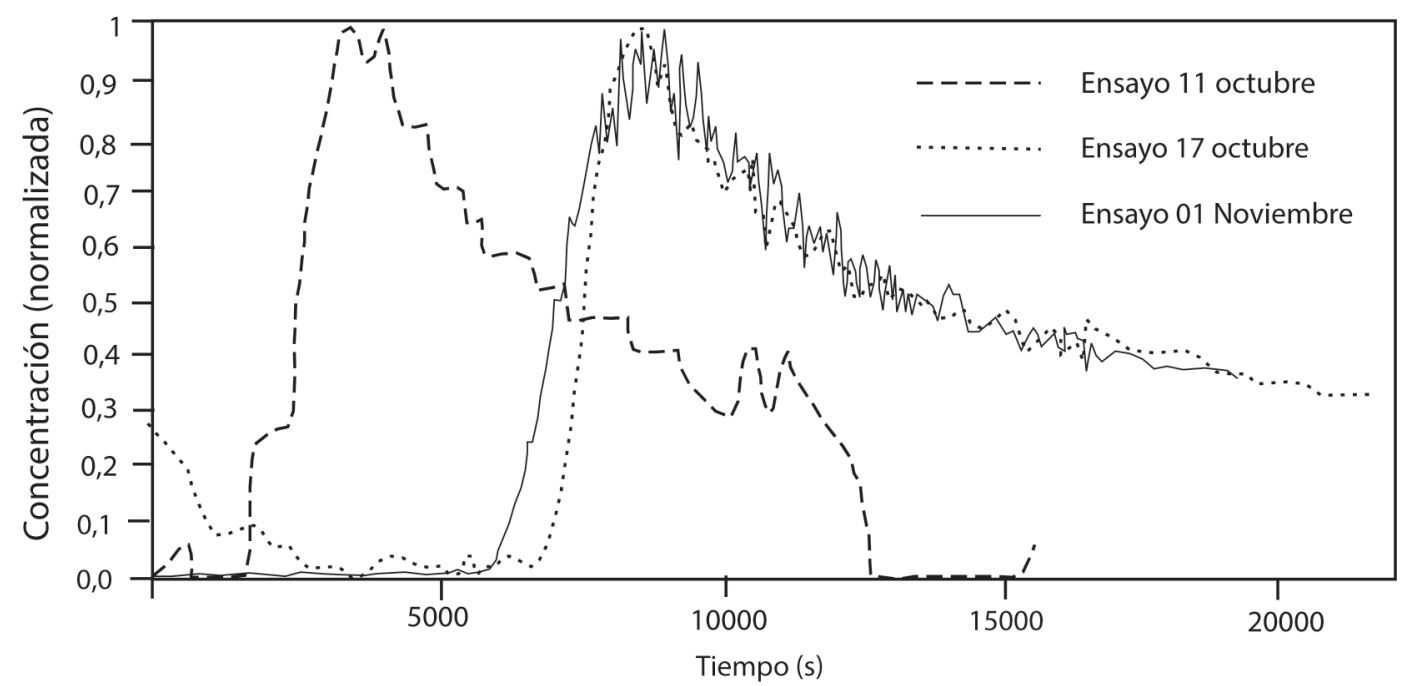

Fig. 5: Curvas de llegada normalizadas a la concentración máxima registrada.

\section{Morfología de las curvas de llegada}

Al normalizar las curvas de llegada con la concentración de pico (Fig. 5) es posible comparar morfológicamente los resultados de los tres ensayos independientemente de la concentración de inyección. En los tres ensayos se observa el primer arribo del trazador seguido de un incremento pronunciado en la concentración hasta alcanzar la zona de pico. Posteriormente, las curvas muestran un decaimiento gradual asimétrico, normalmente denominado como cola de concentración. Este comportamiento asimétrico es típico de sistemas de porosidad simple altamente dispersivos (Sauty, 1980), y de sistemas de doble porosidad o sistemas con fracciones de agua móvil e inmóvil (van Genuchten, 1981). 


\section{SIMULACIÓN DE CURVAS DE LLEGADA A TRAVÉS DE MODELOS ADVECTIVOS- DISPERSIVOS UNIDIMENSIONALES DE POROSIDAD SIMPLE}

Con el objetivo de modelar numéricamente las curvas de llegada, se emplearon las formulaciones para trazadores con inyección instantánea y por pulso mostradas por Kreft \& Zuber (1978) y Lapidus \& Amundson (1952) respectivamente. Se escogieron dichas formulaciones por representar concentraciones en el efluente y no concentraciones residuales, y además por la rapidez con que se inyectó el soluto. Las mediciones realizadas en el manantial San Miguel constituyen concentraciones en el efluente. El resultado obtenido con los modelos de Kreft \& Zuber (1978) y Lapidus \& Amundson (1952), representados en las ecuaciones 1 y 2 , fue normalizado en relación a la concentración pico teórica (predicha por el mismo modelo), con el objetivo de evitar la incertidumbre en el valor de la concentración de inyección especialmente para el Ensayo 3.

Formulación inyección instantánea según modelo de Kreft \& Zuber (1978):

$$
C=\frac{A}{Q} \frac{x}{\sqrt{4 \pi D t^{3}}} \exp \left[-\frac{(x-v t)^{2}}{4 D t}\right]
$$

Donde

$\mathrm{C}=$ concentración simulada por el modelo $\left(\mathrm{M} / \mathrm{L}^{3}\right)$

$\mathrm{A}=$ masa de trazador inyectada $(\mathrm{M})$

$\mathrm{Q}=$ flujo volumétrico del fluido $\left(\mathrm{L}^{3} / \mathrm{T}\right)$

$\mathrm{x}=$ distancia medida desde el punto de inyección $(\mathrm{L})$

$\mathrm{D}=$ dispersión $\left(\mathrm{L}^{2} / \mathrm{T}\right)$

$\mathrm{v}=$ velocidad de poros $\left(\mathrm{L}^{3} / \mathrm{L}^{2} \mathrm{~T}\right)$

$\mathrm{t}=$ tiempo medido desde el momento de la inyección $(\mathrm{T})$

Formulación inyección por pulso según modelo de Lapidus \& Amundson (1952):

$$
C=\left\{\begin{array}{cc}
C_{i}+\left(C_{o}-C_{i}\right) A(x, t) & 0<t \leq t_{o} \\
C_{i}+\left(C_{o}-C_{i}\right) A(x, t)-C_{o} A\left(x, t-t_{o}\right) & t>t o
\end{array}\right.
$$

$$
Q=\left(0,73+0,27 \frac{H-h o}{H}\right) \frac{k x}{2 t}\left(H^{2}-h_{o}{ }^{2}\right)
$$

Donde

$\mathrm{C}=$ concentración simulada por el modelo (M/L3)

$\mathrm{Ci}=$ concentración inicial o de fondo $\left(\mathrm{M} / \mathrm{L}^{3}\right)$

$\mathrm{Co}=$ concentración inyectada $\left(\mathrm{M} / \mathrm{L}^{3}\right)$

$\mathrm{x}=$ distancia medida desde el punto de inyección (L)

$\mathrm{D}=$ dispersión $\left(\mathrm{L}^{2} / \mathrm{T}\right)$

$\mathrm{R}=$ factor de retardo por adsorción en equilibrio (adimensional)

$\mathrm{v}=$ velocidad de poros $\left(\mathrm{L}^{3} / \mathrm{L}^{2} \mathrm{~T}\right)$

$\mathrm{t}=$ tiempo medido desde el inicio de la inyección (T)

to $=$ duración de pulso de inyección $(\mathrm{T})$

erfc $=$ función error complementario

$\mathrm{k}=$ conductividad hidráulica saturada $(\mathrm{L} / \mathrm{T})$

$\mathrm{L}=$ ancho de la zona de extracción

$\mathrm{H}=$ espesor saturado del acuífero medido en el acuífero (L)

$\mathrm{h}=$ elevación del nivel frático en pozo de extracción medido desde la base del acuífero (L)

Para efectos de este trabajo, el parámetro x en las ecuaciones 1 y 2 es la distancia entre el sondeo $37 \mathrm{PHC}$ y el manantial, cuyo valor es $\mathrm{x}=78,8 \mathrm{~m}$. El tiempo $t$ corresponde con los tiempos en que se realizaron las mediciones, y el parámetro to es 20 min según los datos de campo reportados. El factor de retardo $(\mathrm{R})$ se considera con valor unitario por tratarse de un trazador no reactivo. Al haberse corregido los valores de campo por la concentración de fondo, el parámetro Ci de la ecuación 2 toma valor cero. Al normalizar las ecuaciones 1 y 2 con los valores teóricos de concentración pico, los parámetros $\mathrm{A}, \mathrm{Q}$ y $\mathrm{C}_{\mathrm{o}}$ no deben ser considerados de manera explícita en los cálculos. De esta manera los valores desconocidos que deben ser estimados son la velocidad de poros (v) y la dispersión (D). Las ecuaciones 1 y 2 fueron programadas en una hoja electrónica en el programa comercial Microsoft Excel. A través de un ajuste por mínimos cuadrados entre la solución teórica y los valores medidos en campo (ambos normalizados a la concentración 
pico) y ajustando el tiempo de arribo se obtuvo las curvas mostradas en las figuras 6 a 8 .

Las curvas obtenidas muestran un ajuste satisfactorio tanto para la llegada del trazador como para el inicio de la disminución en la concentración. Para ningún caso las formulaciones empleadas permitieron modelar la cola de concentración. Esto indica la presencia de procesos adicionales al transporte advectivo y dispersivo no considerados por estos modelos. En el cuadro 1 se presenta el resumen de los parámetros obtenidos con cada formulación, junto al número de Peclet correspondiente.

Los parámetros obtenidos muestran que el transporte durante el Ensayo 1 estuvo gobernado por procesos dispersivos, esto debido a la mayor velocidad del agua subterránea y por consiguiente mayor caudal, lo que permite acceder a mayor cantidad de caminos de flujo (tanto a través de las fracturas subverticales abiertas como de las inclinadas cerradas). Para el caso del Ensayo 2 el proceso fue dominado por procesos advectivos, y esto se interpreta como producto de la menor velocidad estimada para esa fecha en relación con el Ensayo 1, lo cual genera una concentración del flujo por caminos preferenciales (principalmente en las fracturas subverticales abiertas) y por tanto se reduce la dispersión. En el caso del Ensayo 3 se obtuvo una mayor dispersión en comparación con el Ensayo 2 aún con una velocidad un poco menor. Se interpreta que este comportamiento está regulado por la mayor concentración posiblemente aplicada durante el Ensayo 3, lo que generó un mayor gradiente de concentración longitudinal y por consiguiente un flujo dispersivo más acelerado.

Debido a la leve asimetría observada en las curvas teóricas para los datos del Ensayo 1 (Fig. 6), se procedió a realizar un ejercicio teórico numérico de prueba y error con el objetivo de intentar reproducir la forma de la cola de concentración a partir de la modificación del número de Peclet, sin intención de optimizar los parámetros de transporte o de obtener un significado físico para ellos. Esto es, un ejercicio apenas con el objetivo de reproducir la forma de la curva de campo. En la figura 9 se presenta dicho ajuste para la formulación de Kreft \& Zuber (1978).
Para dicho ajuste se requirió de un valor de Peclet de 0,47 (esto es, alta dispersión) y una velocidad de poros de $302 \mathrm{~m} / \mathrm{d}$. Este bajo número de Peclet genera un arribo casi instantáneo del trazador, por lo que fue necesario aplicar un desplazamiento de 1850 segundos a dicha curva para ajustar el tiempo de arribo observado en campo. Las altas dispersiones podrían explicar la forma asimétrica de la curva de llegada, sin embargo no explicarían el retardo en el tiempo de arribo. Siendo el trazador empleado un trazador conservativo, este retardo no puede ser explicado por procesos de adsorción en equilibrio. De esta manera, procesos adicionales a la advección y dispersión actuaron durante el transporte del trazador.

\section{ESTIMACIÓN DE LA VELOCIDAD DE DARCY Y PROPIEDADES DEL ACUÍFERO}

En este apartado se presenta la estimación de los valores de los parámetros: velocidad de Darcy (vd), permeabilidad saturada (k) y porosidad efectiva $\left(\mathrm{n}_{\mathrm{e}}\right)$ para el acuífero en análisis. Como se indicó en la introducción, en un procedimiento convencional de simulación de transporte de solutos, las características del acuífero deben obtenerse de manera independiente del ensayo del trazador, esto para garantizar que las incógnitas en el proceso de ajuste de las curvas de llegada correspondan solamente a los parámetros de transporte. Debido a que las propiedades del acuífero no fueron caracterizadas explícitamente durante los ensayos de trazador, el procedimiento seguido en este trabajo fue el de aproximar los valores de $\mathrm{vd}, \mathrm{k} \mathrm{y} \mathrm{n}_{\mathrm{e}}$ a partir de las velocidades de poro obtenidas en el ajuste de las curvas de llegada, complementado con los datos de campo disponibles. En el cuadro 2 se resume la información de campo disponible. Solamente para el Ensayo 1 se dispone de información medida para determinar el gradiente hidráulico $(0,033 \mathrm{~m} / \mathrm{m}$ para esa fecha). No se cuenta con la elevación del nivel freático en el sondeo 37 PHC para las fechas de los ensayos 2 y 3.

La naturaleza freática del acuífero y su reacción directa a la recarga por lluvias (como se 


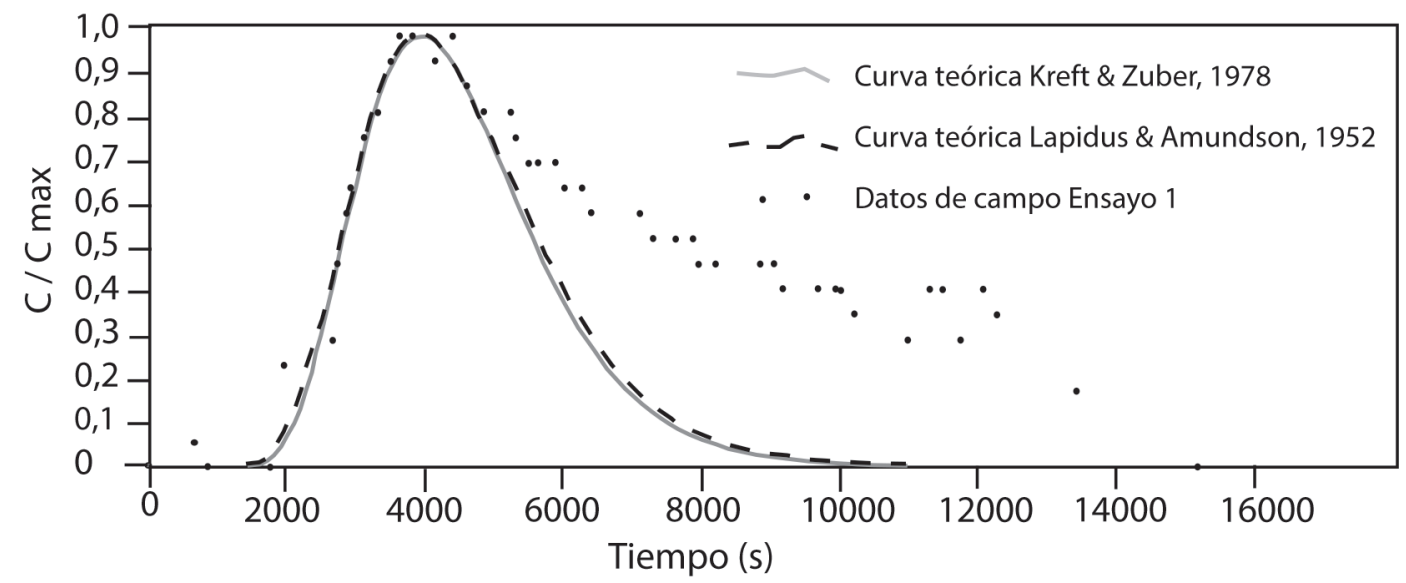

Fig. 6: Simulación de curva de llegada para Ensayo 1.

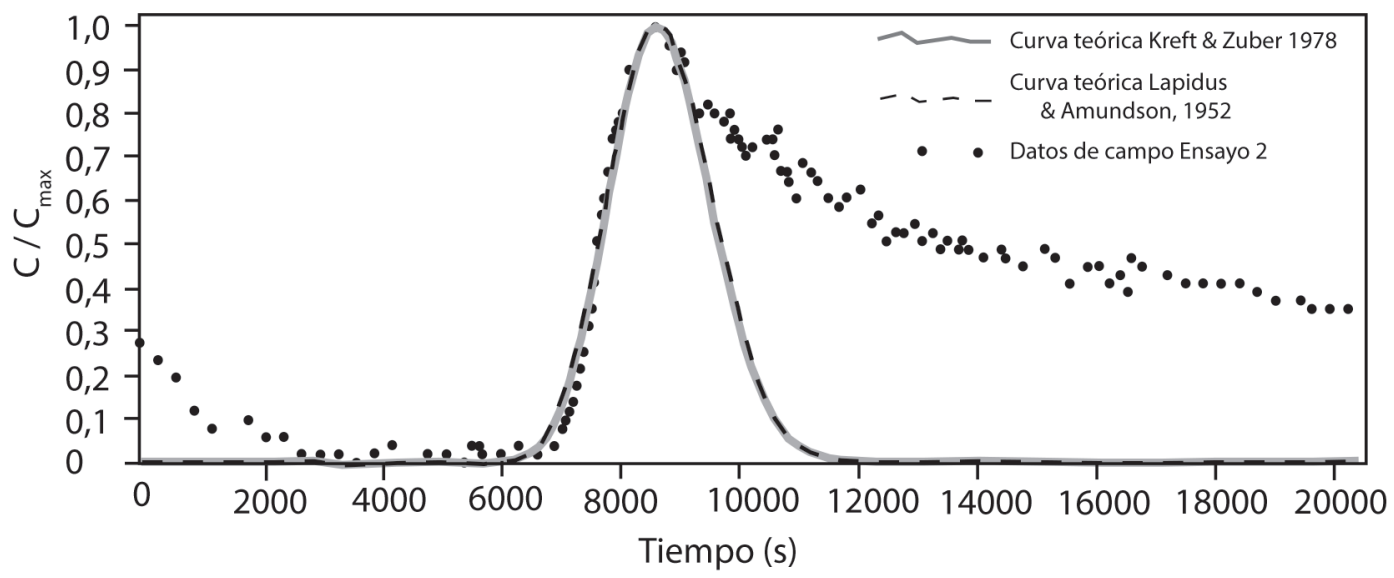

Fig. 7: Simulación de curva de llegada para Ensayo 2.

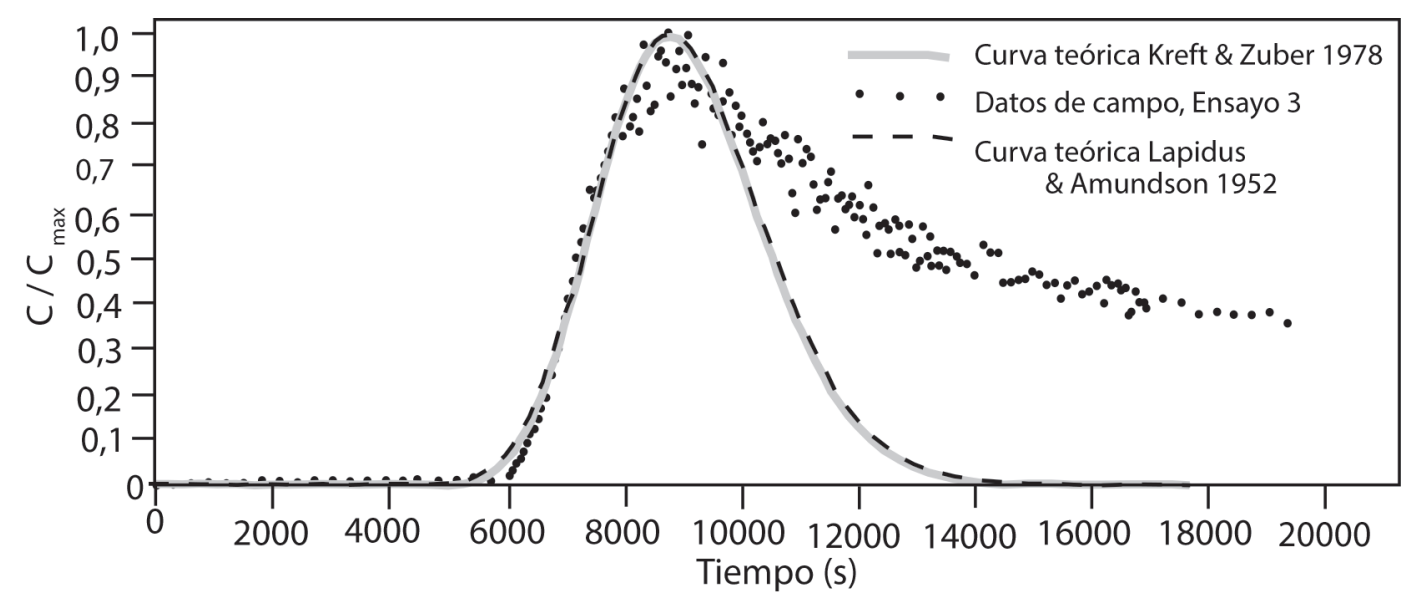

Fig. 8: Simulación de curva de llegada para Ensayo 3. 
Cuadro 1

Parámetros de transporte estimados.

\begin{tabular}{cccccccc}
\hline & \multicolumn{3}{c}{ Kreft \& Zuber (1978) } & \multicolumn{3}{c}{ Lappidus \& Amundson (1952) } \\
\hline Ensayo & Fecha & Veloc. Poros (m/d) & Peclet & Disper. (m) & Veloc. Poros (m/d) & Peclet & Disper (m) \\
\hline 1 & $11 / 10 / 2001$ & 1622 & 21 & 3,75 & 1842 & 14 & 5,63 \\
2 & $17 / 10 / 2001$ & 783 & 235 & 0,34 & 840 & 216 & 0,36 \\
3 & $01 / 11 / 2001$ & 755 & 85 & 0,93 & 807 & 74 & 1,06 \\
\hline
\end{tabular}

muestra más adelante) no permiten asumir que el gradiente hidráulico se mantenga constante durante todo el año; esto implica que el espesor saturado del acuífero puede variar en dependencia de la recarga por lluvias. Por este motivo los gradiente hidráulicos para las fechas de los ensayos 2 y 3 fueron estimados según el procedimiento que se muestra más adelante. La geometría de la naciente es descrita como una sección cuadrada de $2 \mathrm{~m}$ de extensión lateral y por tanto con $4 \mathrm{~m}^{2}$ de área.

El caudal del manantial no fue aforado en las fechas de los ensayos, sin embargo, se cuenta con un registro de aforos de caudal de la Quebrada Naciente, la cual es originada a partir del manantial San Miguel (Fig. 1). En el cuadro 3 se resumen los valores disponibles de caudales aforados para el año 2001.

Los caudales correspondientes a las fechas de ensayo de trazador, fueron estimados a partir de una relación obtenida entre la pluviometría y los caudales aforados. Se cuenta con datos de pluviometría diaria (a partir del 1 de enero de 2001) en la estación Colonia Los Ángeles localizada a $600 \mathrm{~m}$ del manantial San Miguel. En las figuras 10,11 y 12 se presentan los resultados del análisis referido. En la figura 10 se muestra la correlación exponencial existente entre los aforos realizados en las fechas 20 julio a 9 de octubre de 2001 y la pluviosidad acumulada a partir del 29 de mayo.

También se observa en la misma figura una clara tendencia de correlación entre el aumento del caudal y el aumento de la pluviosidad acumulada para los aforos realizados entre 28 de noviembre y 10 de diciembre de 2001 . No existen datos de aforo entre las fechas 9 de octubre y 28 de noviembre de 2001, lapso en el cual se realizaron los tres ensayos. No se tiene documentación de campo para afirmar exactamente en que momento se revierte la tendencia de disminución del caudal observada hasta el 9 de octubre.

En la figura 11 se muestra la relación gráfica entre la pluviometría diaria y los caudales tanto teóricos como aforados. La línea a trazos mostrada en la misma figura corresponde con el caudal teórico para cada día estimado a partir de la ecuación de correlación exponencial de la figura 10.

La discrepancia entre los caudales aforados el 28 de noviembre y 10 de diciembre con la curva teórica, es un claro indicativo de que esta ecuación de correlación presenta una limitación en cuanto

Cuadro 2

Información de campo disponible. ( $\mathrm{S}$ : Si está disponible; N: No está disponible)

\begin{tabular}{cccc}
\hline & Ensayo 1 & Ensayo 2 & Ensayo 3 \\
\hline Elevación de nivel freático en 37 PHC & $\mathrm{S}$ & $\mathrm{N}$ & $\mathrm{N}$ \\
Elevación de nivel freático en naciente & $\mathrm{S}$ & $\mathrm{S}$ & $\mathrm{S}$ \\
Distancia entre naciente y 37 PHC & $\mathrm{S}$ & $\mathrm{S}$ & $\mathrm{S}$ \\
Caudal de naciente & $\mathrm{N}$ & $\mathrm{N}$ & $\mathrm{N}$ \\
Geometría de naciente & $\mathrm{S}$ & $\mathrm{S}$ & $\mathrm{S}$ \\
Pluviometría & $\mathrm{S}$ & $\mathrm{S}$ & $\mathrm{S}$ \\
\hline
\end{tabular}


Cuadro 3

Caudales aforados en la Quebrada Naciente en 2001

\begin{tabular}{cc}
\hline Fecha & $\mathrm{Q}(1 / \mathrm{s})$ \\
\hline $29 / 05 / 2001$ & 17,0 \\
$22 / 06 / 2001$ & 18,0 \\
$20 / 07 / 2001$ & 56,0 \\
$29 / 08 / 2001$ & 31,0 \\
$17 / 09 / 2001$ & 25,0 \\
$09 / 10 / 2001$ & 20,0 \\
$28 / 11 / 2001$ & 62,0 \\
$10 / 12 / 2001$ & 70,0 \\
\hline
\end{tabular}

a la fecha hasta la cual permite una adecuada representación de los caudales, especialmente para el lapso de tiempo entre el 9 de octubre y el 28 de noviembre. Un análisis detallado de la pluviosidad (objeto de una publicación posterior) permitió concluir que el tiempo de respuesta entre los eventos de déficit o superávit de precipitación registrados en la estación Colonia Los Ángeles y los eventos de aumento o disminución de caudal en la quebrada Naciente es en promedio de 21 a 22 días. Esto es, los caudales aforados son el resultado de la acumulación de la variación en la pluviosidad ocurridos hasta 21 a 22 días anteriores a su medición. De esta manera los caudales para las tres fechas de ensayo son el resultado de los eventos de pluviosidad acumulados hasta las fechas 19 de septiembre, 25 de septiembre y 10 de octubre de 2001 respectivamente. El valor de caudal aforado el 9 de octubre de 2001 (2 días antes a la realización del Ensayo 1) es el resultado de los eventos ocurridos hasta el 17 de septiembre de 2001. La variación en el caudal para la fecha del Ensayo 1 responde a las variaciones ocurridas entre el 17 y el 19 de septiembre. En este lapso de tiempo se registró una disminución en la cantidad de lluvia por lo que es de esperar una disminución en el caudal en relación al medido el día 9 de octubre. Para la fecha del Ensayo 2 a pesar de una leve recuperación se aprecia un déficit marcado en la precipitación. Para el periodo correspondiente al Ensayo 3 este déficit aumenta. Es por este motivo que los caudales a esperar son menores a los observados en el aforo del 9 de octubre de 2001.

En la figura 12 se muestra el ajuste polinómico de segundo grado y el coeficiente de correlación para el gráfico de balance de pluviosidad y caudales aforados. El cuadro 4 resume los caudales teóricos estimados para las fechas de los ensayos con base en las dos ecuaciones de correlación mostradas en las figuras 10 y 12 .

Por otro lado, para estimar el valor de la permeabilidad se empleó la ecuación de rebajamiento lineal del nivel freático para acuíferos libres con penetración parcial (ecuación 3) obtenida por Mansur \& Kaufman (1962). Para utilizar esta aproximación se idealizó el manantial San Miguel como un pozo de extracción con penetración parcial al acuífero y el sondeo $37 \mathrm{PHC}$ como el pozo de observación.

$$
Q=\left(0,73+0,27 \frac{H-h o}{H}\right) \frac{k x}{2 L}\left(H^{2}-h_{o}{ }^{2}\right)
$$

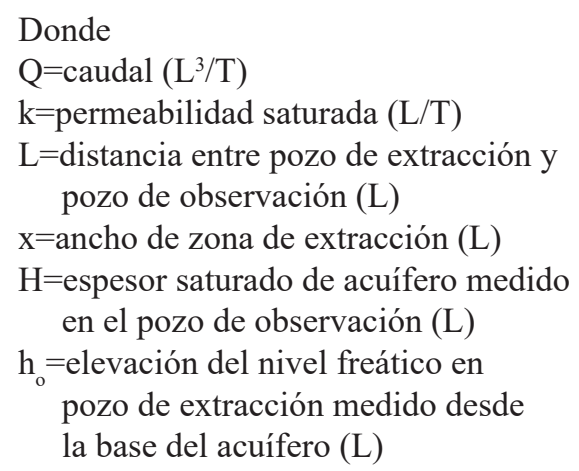

La ecuación 3 fue empleada para los datos del Ensayo 1 con los siguientes parámetros: $\mathrm{Q}=$ $18,997 \mathrm{l} / \mathrm{s}$ y $18,210 \mathrm{l} / \mathrm{s}, \mathrm{L}=78,8 \mathrm{~m}, \mathrm{x}=2 \mathrm{~m}, \mathrm{H}=16,7$ $\mathrm{m} \mathrm{y} \mathrm{h}_{\mathrm{o}}=14,1 \mathrm{~m}$. Los valores de permeabilidad obtenidos para los caudales empleados son: 2005 m/día y $2092 \mathrm{~m} / \mathrm{d}(2,32-2,42 \mathrm{~cm} / \mathrm{s})$. A partir de estos valores de permeabilidad y empleando el gradiente hidráulico de $0,033 \mathrm{~m} / \mathrm{m}$, se estimó la velocidad de vd en el rango 66,17 a $69,03 \mathrm{~m} / \mathrm{d}$. Empleando las velocidades de poros determinadas en el ajuste de curva de llegada (cuadro 1) se obtuvo que la porosidad efectiva del sistema se encuentra en el rango $3,6 \%$ a $4,3 \%$. 


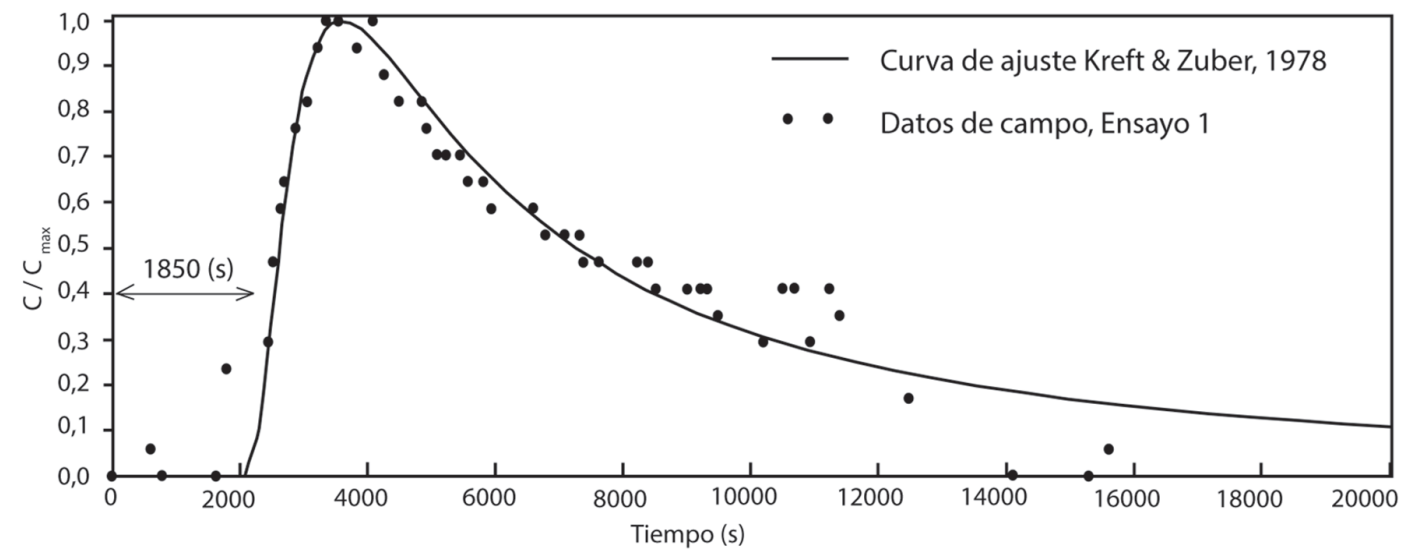

Fig. 9: Ajuste idealizado de curva de llegada para Ensayo 1.

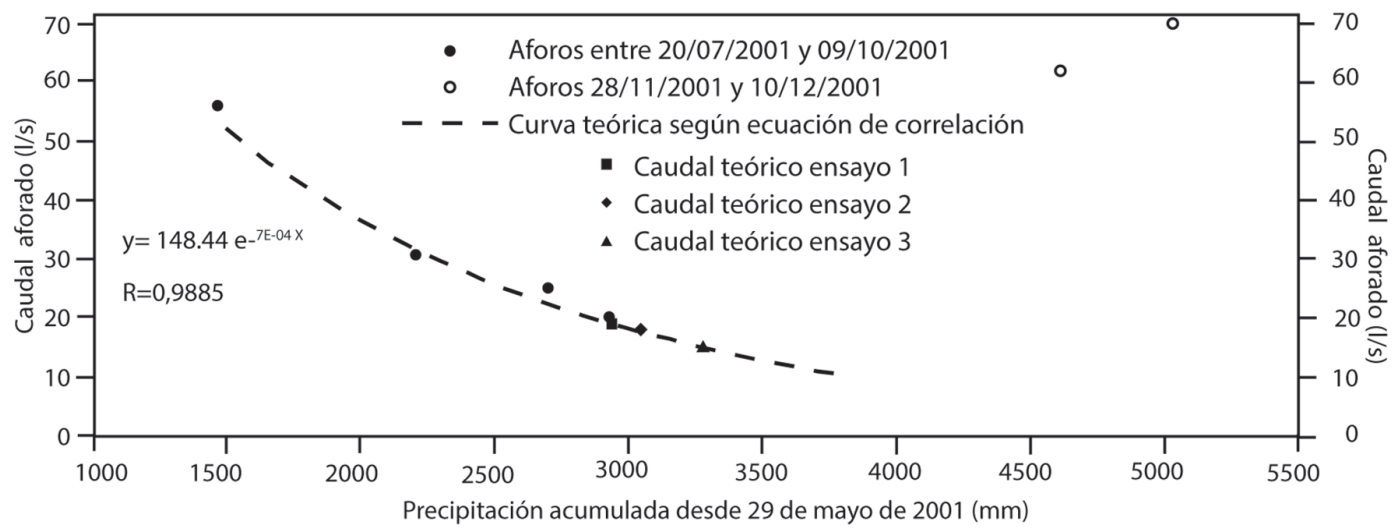

Fig. 10: Correlación entre pluviosidad acumulada y caudal aforado.

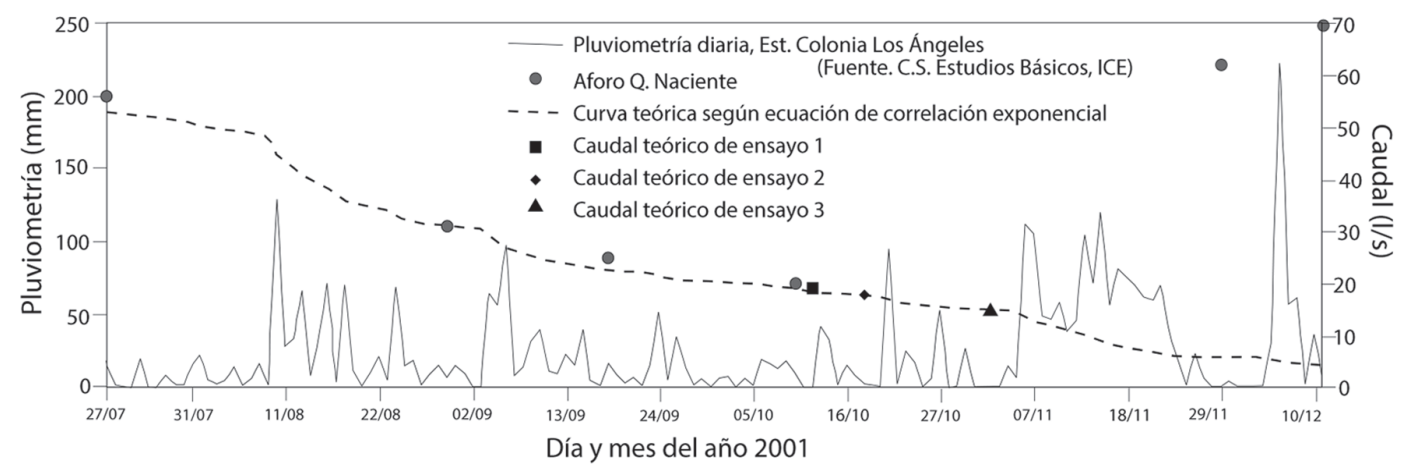

Fig. 11: Relación entre pluviometría y caudales teóricos y aforados. 
Cuadro 4

Caudales teóricos para fechas de ensayos.

$\begin{array}{cccc}\text { Ensayo } & \text { Fecha } & \begin{array}{c}\text { Ajuste } \\ \text { exponencial } \\ (1 / \mathrm{s})\end{array} & \begin{array}{c}\text { Ajuste } \\ \text { polinómico } \\ (1 / \mathrm{s})\end{array} \\ 1 & 11 / 10 / 2001 & 19,0 & 18,2 \\ 2 & 17 / 10 / 2001 & 17,7 & 9,6 \\ 3 & 01 / 11 / 2001 & 15,0 & 7,8\end{array}$

Considerando la permeabilidad y la porosidad como valores constantes en el acuífero, se procedió a estimar los valores de $\mathrm{H}$, para los ensayos 2 y 3 , empleando la ecuación 4 y las velocidades de poros estimadas en la sección anterior (cuadro 1). Con dicho valor de $\mathrm{H}$ se determinó el gradiente hidráulico y con este valor la velocidad de Darcy. Posteriormente con los valores conocidos se procedió a utilizar la ecuación 3 para estimar el caudal de la naciente. El cuadro 5 resume los valores obtenidos para cada ensayo.

$$
v=\frac{k}{n} \frac{\left(H-h_{o}\right)}{L}
$$

Donde

$\mathrm{v}=$ velocidad de poros $(\mathrm{L} / \mathrm{T})$

$\mathrm{k}=$ permeabilidad saturada $(\mathrm{L} / \mathrm{T})$

$\mathrm{L}=$ distancia entre pozo de extracción y pozo de observación (L)

$\mathrm{H}=$ espesor saturado de acuífero medido en el pozo de observación (L)

$\mathrm{h}_{\mathrm{o}}=$ elevación de nivel freático en pozo de extracción medido desde la base del acuíf. (L)
Los valores de caudal estimados a través de este procedimiento están en el mismo rango de los obtenidos con el ajuste polinómico. Se considera pues ese ajuste como el más representativo de las condiciones de caudal para los días de ensayo. Las velocidades vd obtenidas para los ensayos 2 y 3 presentan una diferencia en el orden de $4 \%$, valor bastante cercano al 1,3\% de diferencia observado en los tiempos de llegada de las concentraciones máximas, y dentro del margen de error por tiempos de muestreo.

A manera de comparación, se estimaron las velocidades vd para estos dos ensayos empleando directamente la ecuación 3 con los caudales determinados por el ajuste polinómico. Los valores así obtenidos $37,044 \mathrm{~m} / \mathrm{d}$ y $30,658 \mathrm{~m} / \mathrm{d}$, muestran una diferencia porcentual de $20,8 \%$, mucho mayor a la obtenida con el procedimiento de la ecuación 4. Este resultado demuestra que a pesar del buen ajuste polinómico obtenido, existen imprecisiones en la aproximación que deben ser mejoradas. El caudal estimado por los ajustes exponencial y polinómico para la fecha del Ensayo 1 (cuadro 4) se muestra bastante razonable y eso se debe a la cercanía con el último punto con dato de caudal localizado a solo 2 días de esta fecha.

Los valores de caudal mostrados en el cuadro 5 son más cong ruentes con el retardo en la llegada de las concentraciones de pico de los ensayos 2 y 3 en comparación con el Ensayo 1. De esta manera, el arribo más temprano observado en el Ensayo 1 es producto de una mayor velocidad vd para esa fecha. Los datos corroboran también el hecho de que por corresponder con un acuífero libre con recarga directa por lluvia, las condiciones

Cuadro 5

Velocidad de Darcy (vd), permeabilidad (k) y gradiente hidráulico (i) estimados.

\begin{tabular}{cccccccc}
\hline Ensayo & $\mathrm{k}(\mathrm{m} / \mathrm{d})$ & $\mathrm{n}(\%)$ & $\mathrm{H}(\mathrm{m})$ & $\mathrm{ho}(\mathrm{m})$ & $\mathrm{i}(\mathrm{m} / \mathrm{m})$ & $\mathrm{vd}(\mathrm{m} / \mathrm{d})$ & $\mathrm{Q}(1 / \mathrm{s})$ \\
\hline 1 & $2005-2092$ & $3,6-4,3$ & 16,7 & 14,1 & 0,0330 & $66,17-69,03$ & $18,21-18,99$ \\
2 & $2005-2092$ & $3,6-4,3$ & $15,29-15,36$ & 14,1 & $0,015-0,0159$ & $30,18-33,32$ & $7,7-8,5$ \\
& & & & & $0,0145-$ & $28,99-32,13$ & $7,4-8,2$ \\
3 & $2005-2092$ & $3,6-4,3$ & $15,24-15,31$ & 14,1 & 0,0154 & & \\
\hline
\end{tabular}




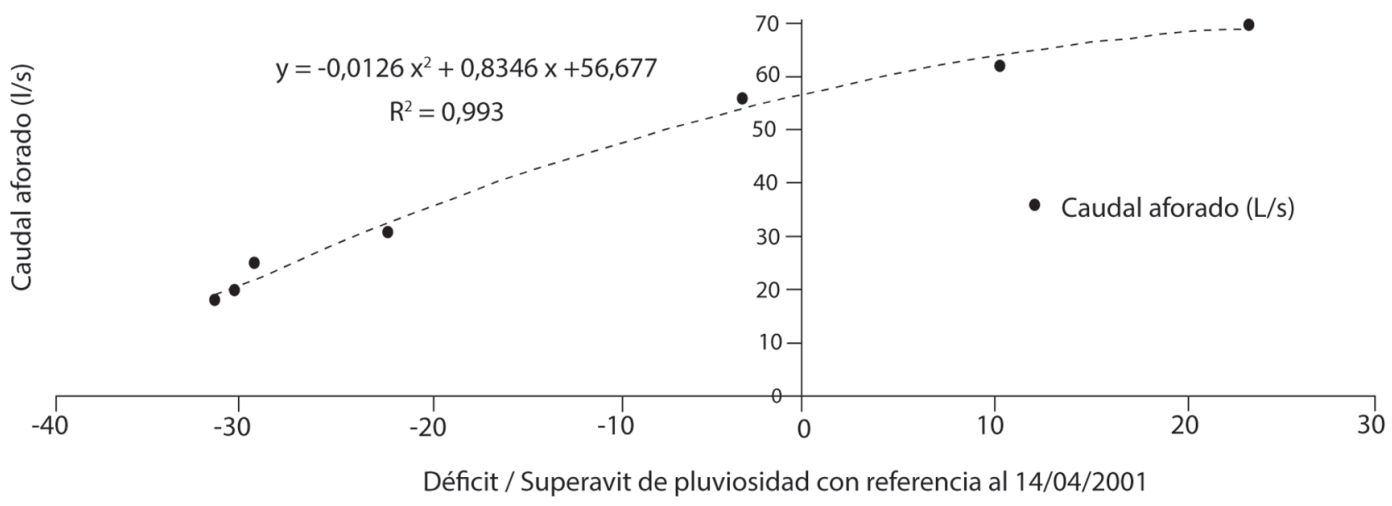

Fig. 12: Ajuste polinómico entre balance de pluviosidad y caudales aforados.

de flujo fueron diferentes para cada día de ensayo en dependencia de las variaciones climatológicas presentadas en los días previos a la realización de los mismos. Debido a que los ensayos se realizaron bajo condiciones de gradiente natural, no se tenía control alguno sobre el flujo, sin embargo esto también demuestra la importancia de realizar caracterizaciones completas del régimen hidráulico cuando se realicen este tipo de ensayos.

Según los datos del cuadro 5, para el Ensayo 3 era de esperarse una velocidad de flujo menor a la del Ensayo 2 y por tanto menor dispersión. Esto parece confirmar el hecho de que la detección más temprana del trazador en el Ensayo 3 es producto de una mayor difusión y no producto de una mayor dispersión mecánica, de acuerdo con los modelos unidimensionales empleados en este análisis.

Los valores de parámetros obtenidos en este trabajo se encuentran dentro del rango de valores reportados en la literatura mundial. Sobre la validación de los parámetros obtenidos se tratará en una publicación posterior.

\section{CONCLUSIONES}

Ensayos de trazadores bajo condiciones de gradiente natural deben realizarse conforme un diseño previo, además deben contar con la mayor cantidad de información posible que permita caracterizar adecuadamente el régimen de flujo actuante.
Esto con el objetivo de restringir la incertidumbre solamente a los parámetros involucrados en el transporte. Debido a la disponibilidad parcial de información de campo, en este trabajo se aplicó un procedimiento no convencional para la estimación tanto de los parámetros de flujo como de transporte a través de la aplicación de modelos advectivos-dispersivos unidimensionales de porosidad simple para inyección instantánea y en pulso. A pesar de las limitaciones en información, los parámetros obtenidos se encuentran dentro del rango de valores reportados en la literatura mundial, y además permitieron explicar de manera adecuada el arribo del trazador al punto de observación, así como el posterior aumento de la concentración hasta el punto de concentración máxima. Los modelos empleados sin embargo no permitieron explicar el decaimiento asimétrico de la concentración posterior al arribo del pico. Esto es, además de los procesos de advección y dispersión, existen procesos adicionales que retardan la llegada del trazador y que no son considerados en los modelos empleados. Estos análisis aunque limitados para simular las formas de las curvas de llegada, permitieron ganar sensibilidad en relación a la magnitud de los efectos de la dispersión y la advección para cada una de las fechas de realización de los ensayos. Los resultados indican que dependiendo de las condiciones naturales de flujo y de las concentraciones de inyección empleadas, el transporte de solutos en este acuífero 
puede pasar de ser dominado por advección a ser dominado por dispersión. De esta manera, durante el Ensayo 1 el mayor caudal estimado (18,219,0 1/s) permitió una mayor dispersión durante el transporte del trazador y un menor tiempo de arribo. Para la fecha del Ensayo 2 las condiciones de caudal en el acuífero fueron menores (7,7-8,5 $1 / \mathrm{s}$ ) concentrando el transporte a través de caminos preferenciales (posiblemente las fracturas subverticales abiertas) y por consiguiente generando menor dispersión en relación al Ensayo 1. Para el Ensayo 3, a pesar de que los caudales estimados son menores al Ensayo 2 (7,4-8,2 1/s), y por consiguiente era de esperar menor dispersión, los resultados de campo y teóricos muestran que durante este ensayo hubo mayor dispersión. Esto se atribuye a la mayor concentración de trazador inyectada durante este ensayo que generó un gradiente de concentración mayor y por consiguiente más transporte por procesos difusivos. Para definir el umbral de velocidad para el cual ocurre la transición entre transporte dominado por advección y transporte dominado por dispersión se requerirá de más ensayos de campo para condiciones variables de flujo o alternativamente un análisis numérico de sensibilidad que incorpore de manera explícita la geometría de las fracturas y su distribución espacial.

Debido a que los ensayos se realizaron bajo condiciones de gradiente natural, no se tenía control alguno sobre el flujo del agua subterránea. Los datos obtenidos en este estudio corroboran el hecho de que por corresponder con un acuífero libre con recarga directa por lluvia, las condiciones de flujo fueron diferentes para cada ensayo en dependencia de las variaciones climatológicas presentadas en los días previos a la realización de los mismos. En caso de realizarse nuevos ensayos con gradiente natural, es muy importante verificar las condiciones de flujo imperantes para ese momento. Los valores de dispersividad obtenidos en este análisis son válidos para estimar el tiempo de arribo y los valores de concentración de pico a esperar en este acuífero para el rango de velocidades determinadas en este estudio. Sin embargo para realizar una caracterización adecuada del tiempo de permanencia del soluto dentro del acuífero será necesario el empleo de modelos de doble porosidad o MIM.

El análisis de los datos de pluviosidad en conjunto con los caudales aforados de la Quebrada Naciente, demuestra la estrecha relación entre la recarga por lluvias y el aporte del acuífero. También demuestran la rápida respuesta del acuífero a los cambios climáticos. Esta observación resalta la importancia de como eventos externos al acuífero como el vertido de contaminantes o grandes sequías pueden verse rápidamente reflejados en los sistemas de captación para consumo humano e industrial. Un análisis detallado de la pluviosidad permitió concluir que el tiempo de respuesta entre los eventos de déficit o superávit de precipitación registrados en la estación Colonia Los Ángeles y los eventos de aumento o disminución de caudal en la quebrada Naciente es en promedio de 21 a 22 días. Durante el año 2001 el manantial San Miguel estuvo caracterizado por un caudal aforado variable entre 17 1/s en la época seca hasta 70 1/s en la época lluviosa. Esta variabilidad se debe al carácter freático del acuífero y a su rápida respuesta a la recarga por lluvias.

Los resultados de este análisis demostraron que el uso de ensayos de trazador es una herramienta útil para entender los procesos de transporte que actúan en un acuífero en particular, y también para obtener valores para los parámetros de transporte. Los tres ensayos aquí analizados indican que además de los procesos advectivos y dispersivos, hay otros procesos que actúan durante el transporte de solutos en acuíferos fracturados, y que estos no pueden ser analizados a través de modelos simples desarrollados para acuíferos de porosidad simple. Para las condiciones centroamericanas donde muchos acuíferos se encuentran en formaciones fracturadas es de esperar un comportamiento similar en las curvas de llegada (sin embargo esto debe corroborarse para cada acuífero) por lo que el empleo de modelos simples puede no ser adecuado para evaluar tanto el potencial de contaminación previo al vertido de cualquier soluto, como la efectividad de las medidas de remediación una vez la contaminación haya ocurrido. 


\section{REFERENCIAS BIBLIOGRÁFICAS}

ALVARADO, G. \& SALANI, F., 2004: Tefroestratigrafia (40 000 - 2000 A.P) en el sector Caribe de los volcanes Barva, Congo y Hule, Cordillera Central, Costa Rica.- Rev. Geol. Amér. Central, 30: 59-72.

CUSTODIO, E. \& LLAMAS, M., 1983: Hidrología subterránea.- 2350 págs. Ediciones Omega. Barcelona.

ESCUDER, R., FRAILE, J., JORDANA, S., RIBERA, F., SÁNCHEZ-VILA, X. \& VÁZQUEZ-SUÑE, E., 2009: Transporte de solutos en aguas subterráneas y ensayos de trazadores.- 768 págs. Edit. Escuder, R., Fraile, J., Jordana, S., Ribera, F., Sánchez-Vila, X. \& Vázquez-Suñé. En Hidrogeología, conceptos básicos de hidrología subterránea.-FCIHS, 378-425.

FETTER, C.W., 1993: Contaminant hydrogeology.- 458 págs. Prentice Hall, Nueva Jersey.

KREFT, A. \& ZUBER, A., 1978: On the physical meaning of the dispersion equation and its solutions for different initial and boundary conditions.- Chem. Engin. Sci. 33: 1471-1480.

LAPIDUS, L. \& AMUNDSON, N. R., 1952: Mathematics of adsorption in beds. VI. The effects of longitudinal diffusion in ion exchange and chromatographic columns.J. Phys. Chem. 56: 984-988.

\section{LOSILLA, M., RODRÍGUEZ,H., SCHOSINSKY,} G., STIMSON, J., BETHUNE, D., 2001: Características hidrogeológicas de la región centroamericana.- En: MORA, R. \& ROJAS, S. (comps): Los acuíferos volcánicos y el desarrollo sostenible en América Central.- Edit. U.C.R., San José: 33-178.
MALOSZEWSKI, P. \& ZUBER, A., 1990: Mathematical modelling of tracer behaviour in short-term experiments in fissured rocks.- Water Resources Res. 26(7): 1517-1528.

MANZUR, C. I. \& KAUFMAN, R. I., 1962: Dewatering. En: Leonards, G.A. (ed.). Foundation Engineering.- Mc Graw - Hill Book Co, 241-350.

PTAK, TH., PIEPENBRINK, M., MARTAC, E., 2004: Tracer test for the investigation of heterogeneous porous media and stochastic modelling of flow and transport- a review of some recent developments.- Journal of hydrology 294 (2004): 122-163.

SAUTY, J.P., 1980: An Analysis of Hydrodispersive Transfer in Aquifers.- Water Resources Research. 16 (1):145-158.

TSANG, D.H., FRIND, E.O., \& SUDICKY, E. A., 1981: Contaminant transport in fractured porous media: analytical solution for a single fracture.- Water Resources Research. 17 (3): 555-564.

VAN GENUCHTEN, M., 1981: Non-Equilibrium transport parameters from miscible displacement experiments.- 88 págs. U.S. Salinity Laboratory. Department of Agriculture Science and Education Administration, California. [Reporte interno de investigación No. 119]

VARGAS, A. \& FERNÁNDEZ, F., 2002: Aspectos hidrogeológicos en el sitio del embalse Cipreses, P.H. Cariblanco y aplicaciones de trazadores en agua subterránea.- Rev. Geól. Amér. Central. 27: 133-142. 
